PROCEEDINGS OF THE

AMERICAN MATHEMATICAL SOCIETY

Volume 128, Number 7, Pages 2017-2031

S 0002-9939(99)05432-5

Article electronically published on November 1, 1999

\title{
OBLIQUE MULTIWAVELETS IN HILBERT SPACES
}

\author{
WAI-SHING TANG \\ (Communicated by David R. Larson)
}

\begin{abstract}
In this paper, we elucidate the relationship between two consecutive levels of a multiresolution in the general setting of a Hilbert space. We first prove a result on an extendability problem and then derive, as a consequence, characterizations of oblique multiwavelets in a Hilbert space.
\end{abstract}

\section{INTRODUCTION}

This is a sequel to our earlier papers $4,7,7,5,10,8$. Here, we again consider wavelet-type problems in a general Hilbert space setting, rather than in the usual concrete case of $L^{2}\left(\mathbf{R}^{d}\right)$, the Hilbert space of square integrable complex-valued functions on $\mathbf{R}^{d}$.

It is well known that one of the major issues in the study of a multiresolution $\left\{V_{n}\right\}$ of $L^{2}\left(\mathbf{R}^{d}\right)$ (see e.g. [3, 6 ) is the relationship between any two consecutive multiresolution spaces $V_{n}$ and $V_{n+1}$ (between only $V_{0}$ and $V_{1}$ in the stationary case). This paper elucidates this relationship in the setting of a Hilbert space.

In 1], wavelet-type objects called oblique multiwavelets in $L^{2}(\mathbf{R})$ are introduced. These are generalizations of biorthogonal multiwavelets and it is noted in 1 that they have more flexible properties. Characterizations of oblique multiwavelets in $L^{2}(\mathbf{R})$ are obtained in [1] and 2], using the machinery of the Fourier transform on $L^{2}(\mathbf{R})$. This paper extends these characterizations to the general setting of oblique multiwavelets in a Hilbert space $H$, where the Fourier transform is no longer available and the roles of the translation operator and the dilation operator on $L^{2}(\mathbf{R})$ are replaced by certain unitary operators on $H$. Indeed, we first prove a result on a related extendability problem and then obtain, as a consequence, the above results on oblique multiwavelets in a Hilbert space.

Throughout this paper, $H$ denotes a complex Hilbert space. A sequence $\left\{v_{n}\right\}$ in $H$ is a Riesz basis for its closed linear span $\overline{\operatorname{span}}\left\{v_{n}\right\}$ if there exist positive constants $A$ and $B$ such that

$$
A \sum\left|a_{n}\right|^{2} \leq\left\|\sum a_{n} v_{n}\right\|^{2} \leq B \sum\left|a_{n}\right|^{2}, \quad \forall\left\{a_{n}\right\} \in \ell^{2} .
$$

Two sequences $\left\{v_{n}\right\}$ and $\left\{\tilde{v}_{n}\right\}$ in $H$ are biorthogonal if

$$
\left\langle v_{n}, \tilde{v}_{m}\right\rangle=\delta_{n, m} \quad \forall n, m,
$$

where $\langle x, y\rangle$ denotes the inner product of two vectors $x$ and $y$ in $H$. (See [11].)

Received by the editors August 24, 1998.

2000 Mathematics Subject Classification. Primary 46C99, 47B99, 46B15.

Key words and phrases. Riesz basis, biorthogonal system, oblique projection, multiwavelets.

(C)2000 American Mathematical Society 
Let $U=\left(U_{1}, \ldots, U_{d}\right)$ be an ordered $d$-tuple of distinct unitary operators on a Hilbert space $H$ such that $U_{k} U_{j}=U_{j} U_{k}, k, j=1, \ldots, d$. We shall use the multiindex notation $U^{m}=U_{1}^{m_{1}} \cdots U_{d}^{m_{d}}$ for $m=\left(m_{1}, \ldots, m_{d}\right) \in \mathbf{Z}^{d}$, with the convention that $U_{j}^{0}$ is the identity operator on $H, j=1, \ldots, d$. We also assume that $U^{m}$ is the identity operator only if $m=0$.

For a subset $S$ of $H$, let $\langle S\rangle$ denote the closed linear span of $S$, and

$$
U^{\mathbf{Z}^{d}}(S):=\left\{U^{n} s: n \in \mathbf{Z}^{d}, s \in S\right\} .
$$

If $V=\left\{v_{1}, \ldots, v_{r}\right\}$ and $W=\left\{w_{1}, \ldots, w_{p}\right\}$ are finite subsets of $H$ such that

$$
\left\{\left\langle v_{k}, U^{n} w_{j}\right\rangle\right\}_{n \in \mathbf{Z}^{d}} \in \ell^{2}\left(\mathbf{Z}^{d}\right), \quad k=1, \ldots, r, j=1, \ldots, p,
$$

then the function $\Phi_{V, W}$ defined almost everywhere on $\mathbf{R}^{d}$ by

$$
\Phi_{V, W}(u):=\left(\sum_{n \in \mathbf{Z}^{d}}\left\langle v_{k}, U^{n} w_{j}\right\rangle e^{i n \cdot u}\right)_{1 \leq k \leq r, 1 \leq j \leq p}
$$

is in $L^{2}\left(\mathbf{T}^{d}\right)^{r \times p}$, the space of all $r \times p$ matrices with entries in $L^{2}\left(\mathbf{T}^{d}\right)$ (often identified with $L^{2}\left(\mathbf{T}^{d}, \mathbf{C}^{r \times p}\right)$, where $\mathbf{C}^{r \times p}$ is the space of all $r \times p$ complex matrices equipped with the operator norm, or depending on the context, with the Frobenius norm). For simplicity, we write $L^{2}\left(\mathbf{T}^{d}\right)^{p}$ for $L^{2}\left(\mathbf{T}^{d}\right)^{1 \times p}$.

If $V$ and $W$ are closed linear subspaces of $H$ such that $V \cap W=\{0\}$ and the vector sum $V_{1}=V+W$ is closed, then we write $V_{1}=V \oplus W$ and call this a direct sum. In this case, we define the maps $P_{V / / W}$ and $P_{W / / V}$ from $V_{1}$ to $V_{1}$ by

$$
P_{V / / W}(v+w)=v, \quad P_{W / / V}(v+w)=w, \quad v \in V, w \in W,
$$

and call $P_{V / / W}$ the (oblique) projection of $V_{1}$ on $V$ along $W$ and $P_{W / / V}$ the (oblique) projection of $V_{1}$ on $W$ along $V$. For the special case when $W=V_{1} \cap V^{\perp}$, the orthogonal complement of $V$ in $V_{1}$, we write $V_{1}=V \oplus^{\perp} W$ for the orthogonal direct sum.

We now give a summary of the contents of this paper. In section 2, we obtain necessary and sufficient conditions for the existence of solutions to an extendability problem in a Hilbert space that is related to multiresolution. Section 3 summarizes some useful results on the connection between $L^{2}\left(\mathbf{T}^{d}\right)$ and dilation matrices. The final section elucidates the relationship between two consecutive levels of a multiresolution-type structure and derives characterizations of oblique multiwavelets in a Hilbert space.

\section{An EXTENDABILITY PROBLEM}

Throughout this section, let $Y=\left\{y_{1}, \ldots, y_{s}\right\}$ and $\tilde{Y}=\left\{\tilde{y}_{1}, \ldots, \tilde{y}_{s}\right\}$ be finite subsets of $H$ such that $U^{\mathbf{Z}^{d}}(Y)$ and $U^{\mathbf{Z}^{d}}(\tilde{Y})$ are biorthogonal Riesz bases for $V_{1}=\left\langle U^{\mathbf{Z}^{d}}(Y)\right\rangle=\left\langle U^{\mathbf{Z}^{d}}(\widetilde{Y})\right\rangle$. Moreover, let $X=\left\{x_{1}, \ldots, x_{r}\right\}$ be a finite subset of $H$, where $r<s$, let $U^{\mathbf{Z}^{d}}(X)$ be a Riesz basis for its closed linear span $V_{0}$ and let

$$
V_{0} \subset V_{1} .
$$

In this case, we have the biorthogonal expansions

$$
f=\sum_{j=1}^{s} \sum_{n \in \mathbf{Z}^{d}}\left\langle f, U^{n} \tilde{y}_{j}\right\rangle U^{n} y_{j}=\sum_{j=1}^{s} \sum_{n \in \mathbf{Z}^{d}}\left\langle f, U^{n} y_{j}\right\rangle U^{n} \tilde{y}_{j}, \quad f \in V_{1},
$$




$$
\Phi_{Y, \widetilde{Y}}=\Phi_{\widetilde{Y}, Y}=I_{s} \text { a.e. },
$$

where $I_{s}$ is the $s \times s$ identity matrix, and the entries of the matrix functions $\Phi_{V, Y}$ and $\Phi_{V, \tilde{Y}}$ are $L^{2}$-functions for any finite subset $V$ of $H$.

Theorem 2.1. Let $\Gamma=\left\{z_{1}, \ldots, z_{s-r}\right\}$ be a subset of $V_{1} \backslash V_{0}$ and let $S=X \cup \Gamma$. The following conditions are equivalent:

(i) $U^{\mathbf{Z}^{d}}(S)$ is a Riesz basis for $V_{1}$.

(ii) $U^{\mathbf{Z}^{d}}(\Gamma)$ is a Riesz basis for $W_{0}:=\left\langle U^{\mathbf{Z}^{d}}(\Gamma)\right\rangle$ and $V_{0} \oplus W_{0}=V_{1}$.

(iii) There exist positive constants $C_{1}$ and $C_{2}$ such that

$$
C_{1} \leq \Phi_{S, \widetilde{Y}}(u) \Phi_{S, \widetilde{Y}}(u)^{*} \leq C_{2} \quad \text { a.e. }
$$

(iv) The matrices $\Phi_{S, \widetilde{Y}}(u)$ are invertible for almost all $u$, and the functions $u \longrightarrow$ $\left\|\Phi_{S, \widetilde{Y}}(u)\right\|$ and $u \longrightarrow\left\|\Phi_{S, \widetilde{Y}}(u)^{-1}\right\|$ are essentially bounded.

(v) The operator $R_{0}: L^{2}\left(\mathbf{T}^{d}\right)^{r} \oplus L^{2}\left(\mathbf{T}^{d}\right)^{s-r} \longrightarrow L^{2}\left(\mathbf{T}^{d}\right)^{s}$ defined by

$$
\begin{aligned}
R_{0}(A, B)(u) & =(A(u) B(u))\left(\begin{array}{c}
\Phi_{X, \widetilde{Y}}(u) \\
\Phi_{\Gamma, \widetilde{Y}}(u)
\end{array}\right) \\
& =A(u) \Phi_{X, \widetilde{Y}}(u)+B(u) \Phi_{\Gamma, \widetilde{Y}}(u)
\end{aligned}
$$

is bounded and invertible.

Proof. (i) $\Longrightarrow$ (iii) follows from [10, Proposition 3.4].

(iii) $\Longrightarrow$ (ii): Again by [10. Proposition 3.4], $U^{\mathbf{Z}^{d}}(S)$ is a Riesz basis for $\left\langle U^{\mathbf{Z}^{d}}(S)\right\rangle$, which is a subset of $V_{1}$. In particular, the set $S$ is linearly independent, $U^{\mathbf{Z}^{d}}(\Gamma)$ is a Riesz basis for $W_{0}:=\left\langle U^{\mathbf{Z}^{d}}(\Gamma)\right\rangle$ and

$$
V_{0} \oplus W_{0}=\left\langle U^{\mathbf{Z}^{d}}(S)\right\rangle \subset V_{1}=\left\langle U^{\mathbf{Z}^{d}}(Y)\right\rangle .
$$

Since $\#(S)=s=\#(Y)$, by [5, Theorem 2.4], $\left\langle U^{\mathbf{Z}^{d}}(S)\right\rangle=V_{1}$.

By [10. Theorem 2.1], we have (ii) $\Longrightarrow$ (i). Finally, (iii) $\Longleftrightarrow($ iv) follows from standard arguments in operator theory, and (iv) $\Longleftrightarrow(\mathrm{v})$ follows from [9] pp. 351$352]$.

It is shown in [5, Theorem 2.5] ( 7 , Corollary 3.4] for the case $d=1$ ) that there exist $z_{1}, \ldots, z_{s-r}$ in $V_{1} \cap V_{0}^{\perp}$ such that condition (ii) in Theorem 2.1 holds. In the more general setting of a pair of biorthogonal multiresolutions, [10, Theorem 3.6] shows that again there exist $z_{1}, \ldots, z_{s-r}$ satisfying Theorem[2.1 (ii) and some other conditions.

Proposition 2.2. Suppose that the conditions in Theorem 2.1 hold. Let $\widetilde{S}$ be a subset of $V_{1}$ such that $U^{\mathbf{Z}^{d}}(\widetilde{S})$ is a Riesz basis for $V_{1}$ biorthogonal to $U^{\mathbf{Z}^{d}}(S)$. Write $\widetilde{S}=X^{\prime} \cup \Gamma^{\prime}$ such that $\#\left(X^{\prime}\right)=r, \#\left(\Gamma^{\prime}\right)=s-r, U^{\mathbf{Z}^{d}}\left(X^{\prime}\right)$ biorthogonal to $U^{\mathbf{Z}^{d}}(X)$, $U^{\mathbf{Z}^{d}}\left(\Gamma^{\prime}\right)$ biorthogonal to $U^{\mathbf{Z}^{d}}(\Gamma), U^{\mathbf{Z}^{d}}\left(X^{\prime}\right) \perp U^{\mathbf{Z}^{d}}(\Gamma)$ and $U^{\mathbf{Z}^{d}}\left(\Gamma^{\prime}\right) \perp U^{\mathbf{Z}^{d}}(X)$. Then $R_{0}^{-1}: L^{2}\left(\mathbf{T}^{d}\right)^{s} \longrightarrow L^{2}\left(\mathbf{T}^{d}\right)^{r} \oplus L^{2}\left(\mathbf{T}^{d}\right)^{s-r}$ is given by

$$
R_{0}^{-1}(C)(u)=\left(C(u) \Phi_{X^{\prime}, Y}(u)^{*}, C(u) \Phi_{\Gamma^{\prime}, Y}(u)^{*}\right) .
$$

Proof. By [5. Theorem 2.4], $\Phi_{S, \widetilde{Y}}(u)^{-1}=\Phi_{\widetilde{S}, Y}(u)^{*}=\left(\Phi_{X^{\prime}, Y}(u)^{*} \Phi_{\Gamma^{\prime}, Y}(u)^{*}\right)$. By Theorem 2.1$] R_{0}^{-1}(C)(u)=C(u) \Phi_{S, \tilde{Y}}(u)^{-1}=\left(C(u) \Phi_{X^{\prime}, Y}(u)^{*} \quad C(u) \Phi_{\Gamma^{\prime}, Y}(u)^{*}\right)$. 
Remark 2.3. (i) Under the assumptions of Proposition 2.2 let $V_{0}^{\prime}=\left\langle U^{\mathbf{Z}^{d}}\left(X^{\prime}\right)\right\rangle$ and $W_{0}^{\prime}=\left\langle U^{\mathbf{Z}^{d}}\left(\Gamma^{\prime}\right)\right\rangle$. Then we arrive at the biorthogonal setting

$$
V_{0} \oplus W_{0}=V_{1}, \quad V_{0}^{\prime} \oplus W_{0}^{\prime}=V_{1}, \quad V_{0} \perp W_{0}^{\prime}, \quad V_{0}^{\prime} \perp W_{0} .
$$

Note that $V_{0}^{\prime}$ may not necessarily equal $V_{0}$ and $W_{0}^{\prime}$ may not necessarily equal $W_{0}$.

(ii) If moreover $U^{\mathbf{Z}^{d}}(X) \perp U^{\mathbf{Z}^{d}}(\Gamma)$, then $V_{0}^{\prime}=V_{0}$ and $W_{0}^{\prime}=W_{0}$, and we arrive at the semiorthogonal setting $V_{0} \oplus^{\perp} W_{0}=V_{1}$.

Theorem 2.4. Suppose that the conditions in Theorem 2.1 hold. Let

$$
\begin{aligned}
& A_{j}(u)=\sum_{n \in \mathbf{Z}^{d}} a_{j}(n) e^{i n \cdot u}, \quad a_{j} \in \ell^{2}\left(\mathbf{Z}^{d}\right), \quad j=1, \ldots, r, \\
& B_{k}(u)=\sum_{n \in \mathbf{Z}^{d}} b_{k}(n) e^{i n \cdot u}, \quad b_{k} \in \ell^{2}\left(\mathbf{Z}^{d}\right), \quad k=1, \ldots, s-r, \\
& C_{\ell}(u)=\sum_{n \in \mathbf{Z}^{d}} c_{\ell}(n) e^{i n \cdot u}, \quad c_{\ell} \in \ell^{2}\left(\mathbf{Z}^{d}\right), \quad \ell=1, \ldots, s,
\end{aligned}
$$

and

$$
A=\left(A_{1} \ldots A_{r}\right), \quad B=\left(B_{1} \ldots B_{s-r}\right), C=\left(C_{1} \ldots C_{s}\right) .
$$

The following conditions are equivalent:

$$
\sum_{\ell=1}^{s} \sum_{n \in \mathbf{Z}^{d}} c_{\ell}(n) U^{n} y_{\ell}=\sum_{j=1}^{r} \sum_{n \in \mathbf{Z}^{d}} a_{j}(n) U^{n} x_{j}+\sum_{k=1}^{s-r} \sum_{n \in \mathbf{Z}^{d}} b_{k}(n) U^{n} z_{k} .
$$

$$
\text { (Reconstruction algorithm) }
$$

$$
\begin{aligned}
C(u) & =R_{0}(A, B)(u) \\
& =A(u) \Phi_{X, \widetilde{Y}}(u)+B(u) \Phi_{\Gamma, \widetilde{Y}}(u) .
\end{aligned}
$$

(iii) (Decomposition algorithm)

$$
\begin{aligned}
(A(u), B(u)) & =R_{0}^{-1}(C)(u), \text { i.e. } \\
A(u) & =C(u) \Phi_{X^{\prime}, Y}(u)^{*} \\
B(u) & =C(u) \Phi_{\Gamma^{\prime}, Y}(u)^{*} .
\end{aligned}
$$

Proof. (i) $\Longrightarrow$ (ii): Suppose that (i) holds. Write (i) as $y=x+z$ such that $y \in$ $V_{1}, x \in V_{0}$ and $z \in W_{0}$. Then by [10, Proposition 3.3],

$$
\begin{aligned}
C(u) & =\Phi_{\{y\}, \widetilde{Y}}(u)=\Phi_{\{y\}, \widetilde{S}}(u) \Phi_{\widetilde{Y}, S}(u)^{*} \\
& =\left(\Phi_{\{y\}, X^{\prime}}(u) \Phi_{\{y\}, \Gamma^{\prime}}(u)\right) \Phi_{S, \widetilde{Y}}(u) \\
& =\left(\Phi_{\{x\}, X^{\prime}}(u) \Phi_{\{z\}, \Gamma^{\prime}}(u)\right) \Phi_{S, \widetilde{Y}}(u) \\
& =(A(u) B(u)) \Phi_{S, \tilde{Y}}(u),
\end{aligned}
$$

since $z \perp U^{\mathbf{Z}^{d}}\left(X^{\prime}\right)$ and $x \perp U^{\mathbf{Z}^{d}}\left(\Gamma^{\prime}\right)$. Hence (ii) holds.

The proof of (ii) $\Longleftrightarrow$ (iii) is obvious. Suppose that (ii) holds. Let

$$
x=\sum_{j=1}^{r} \sum_{n \in \mathbf{Z}^{d}} a_{j}(n) U^{n} x_{j} \quad \text { and } \quad z=\sum_{k=1}^{s-r} \sum_{n \in \mathbf{Z}^{d}} b_{k}(n) U^{n} z_{k} .
$$

Let $y=x+z$ be expressed as $\sum_{\ell=1}^{s} \sum_{n \in \mathbf{Z}^{d}} c_{\ell}^{\prime}(n) U^{n} y_{\ell}$, where $c_{\ell}^{\prime} \in \ell^{2}\left(\mathbf{Z}^{d}\right), \quad \ell=$ $1, \ldots, s$. If $C_{\ell}^{\prime}(u)=\sum_{n \in \mathbf{Z}^{d}} c_{\ell}^{\prime}(n) e^{i n \cdot u}, \ell=1, \ldots, s$, then by the implication (i) $\Longrightarrow$ (ii) 
established above and (ii), $\left(C_{1}^{\prime}(u) \ldots C_{s}^{\prime}(u)\right)=(A(u) B(u)) \Phi_{S, \widetilde{Y}}(u)=C(u)$. Hence $c_{\ell}^{\prime}=c_{\ell}, \ell=1, \ldots, s$, and so (i) holds.

Corollary 2.5. Suppose that the conditions in Theorem [2.1 hold. If

$$
y=\sum_{\ell=1}^{s} \sum_{n \in \mathbf{Z}^{d}} c_{\ell}(n) U^{n} y_{\ell}
$$

and

$$
C(u)=\left(\sum_{n \in \mathbf{Z}^{d}} c_{1}(n) e^{i n \cdot u} \ldots \sum_{n \in \mathbf{Z}^{d}} c_{s}(n) e^{i n \cdot u}\right)
$$

then

$$
P_{V_{0} / / W_{0}}(y)=\sum_{j=1}^{r} \sum_{n \in \mathbf{Z}^{d}} a_{j}(n) U^{n} x_{j} \quad \text { and } \quad P_{W_{0} / / V_{0}}(y)=\sum_{k=1}^{s-r} \sum_{n \in \mathbf{Z}^{d}} b_{k}(n) U^{n} z_{k},
$$

where

$$
\left(\sum_{n \in \mathbf{Z}^{d}} a_{1}(n) e^{i n \cdot u} \cdots \sum_{n \in \mathbf{Z}^{d}} a_{r}(n) e^{i n \cdot u}\right)=C(u) \Phi_{X^{\prime}, Y}(u)^{*}
$$

and

$$
\left(\sum_{n \in \mathbf{Z}^{d}} b_{1}(n) e^{i n \cdot u} \ldots \sum_{n \in \mathbf{Z}^{d}} b_{s-r}(n) e^{i n \cdot u}\right)=C(u) \Phi_{\Gamma^{\prime}, Y}(u)^{*}
$$

\section{Some Connections Between $L^{2}\left(\mathbf{T}^{d}\right)$ AND Dilation Matrices}

For the rest of this paper, let $M$ be a $d \times d$ matrix with integer entries such that $m=|\operatorname{det}(M)| \geq 2$. Such a matrix is often called a dilation matrix. Let $M^{T}$ be the transpose of $M$. Let $\mathcal{C}_{M}=\left\{\gamma_{0}, \gamma_{1}, \ldots, \gamma_{m-1}\right\}$ (respectively $\mathcal{C}_{M^{T}}=$ $\left\{\eta_{0}, \eta_{1}, \ldots, \eta_{m-1}\right\}$ ) be a full set of coset representatives of $\mathbf{Z}^{d} / M \mathbf{Z}^{d}$ (respectively $\left.\mathbf{Z}^{d} / M^{T} \mathbf{Z}^{d}\right)$, where $\gamma_{0}=\eta_{0}=0$. Then $\mathbf{Z}^{d}$ is the disjoint union of the cosets $M \mathbf{Z}^{d}+\gamma_{j}, j=0,1, \ldots, m-1$ (respectively $M^{T} \mathbf{Z}^{d}+\eta_{j}, j=0,1, \ldots, m-1$ ).

It is well known that the following orthogonality relations hold:

$$
\sum_{k=0}^{m-1} e^{i 2 \pi\left(\eta_{j}-\eta_{\ell}\right) \cdot M^{-1} \gamma_{k}}=m \delta_{j, \ell}, \quad j, \ell=0,1, \ldots, m-1,
$$

and

$$
\sum_{j=0}^{m-1} e^{i 2 \pi \eta_{j} \cdot M^{-1}\left(\gamma_{k}-\gamma_{\ell}\right)}=m \delta_{k, \ell}, \quad k, \ell=0,1, \ldots, m-1 .
$$

Let

$$
Q(u)=\frac{1}{\sqrt{m}}\left(e^{i \gamma_{k} \cdot u} e^{i 2 \pi \eta_{\ell} \cdot M^{-1} \gamma_{k}} I_{r}\right)_{k, \ell=0}^{m-1}, \quad u \in \mathbf{R}^{d},
$$

where $I_{r}$ is the $r \times r$ identity matrix. By (3.1) and (3.2), $Q(u)$ is a unitary $m r \times m r$ matrix (indeed the product of two unitary matrices). 
Let $q$ be a fixed positive integer and let the map

$$
J: \underbrace{L^{2}\left(\mathbf{T}^{d}\right)^{q \times r} \oplus \ldots \oplus L^{2}\left(\mathbf{T}^{d}\right)^{q \times r}}_{m \text { times }} \longrightarrow L^{2}\left(\mathbf{T}^{d}\right)^{q \times r}
$$

be defined by

$$
\begin{aligned}
& J\left(G_{0}, \ldots, G_{m-1}\right)(u)=\left(G_{0}\left(M^{T} u\right) \ldots G_{m-1}\left(M^{T} u\right)\right) \Omega(u) \\
& =G_{0}\left(M^{T} u\right)+G_{1}\left(M^{T} u\right) e^{i \gamma_{1} \cdot u}+\ldots+G_{m-1}\left(M^{T} u\right) e^{i \gamma_{m-1} \cdot u} \\
& =\sum_{j=0}^{m-1} \sum_{n \in \mathbf{Z}^{d}} g_{j}(n) e^{i\left(M n+\gamma_{j}\right) \cdot u}
\end{aligned}
$$

where $G_{j}(u)=\sum_{n \in \mathbf{Z}^{d}} g_{j}(n) e^{i n \cdot u}, \quad g_{j} \in \ell^{2}\left(\mathbf{Z}^{d}\right)^{q \times r}, \quad j=0,1, \ldots, m-1$, and

$$
\Omega(u)=\left(\begin{array}{c}
I_{r} \\
e^{i \gamma_{1} \cdot u} I_{r} \\
\vdots \\
e^{i \gamma_{m-1} \cdot u} I_{r}
\end{array}\right), \quad u \in \mathbf{R}^{d} .
$$

Theorem 3.1. The map $J$ is a unitary operator, and its inverse

$$
J^{*}: L^{2}\left(\mathbf{T}^{d}\right)^{q \times r} \longrightarrow \underbrace{L^{2}\left(\mathbf{T}^{d}\right)^{q \times r} \oplus \ldots \oplus L^{2}\left(\mathbf{T}^{d}\right)^{q \times r}}_{m \text { times }}
$$

is given by $J^{*}(G)(u)=\left(F_{0}(u), \ldots, F_{m-1}(u)\right)$, where

$$
G(u)=\sum_{n \in \mathbf{Z}^{d}} g(n) e^{i n \cdot u}, \quad F_{j}(u)=\sum_{n \in \mathbf{Z}^{d}} g\left(M n+\gamma_{j}\right) e^{i n \cdot u}, \quad j=0,1, \ldots, m-1 .
$$

Proof. Since

$$
\left\|J\left(G_{0}, \ldots, G_{m-1}\right)\right\|^{2}=\sum_{j=0}^{m-1} \sum_{n \in \mathbf{Z}^{d}}\left\|g_{j}(n)\right\|^{2}=\sum_{j=0}^{m-1}\left\|G_{j}\right\|^{2},
$$

the map $J$ is isometric. For any $G$ in $L^{2}\left(\mathbf{T}^{d}\right)^{q \times r}$,

$$
\begin{aligned}
G(u) & =\sum_{n \in \mathbf{Z}^{d}} g(n) e^{i n \cdot u}=\sum_{j=0}^{m-1} \sum_{n \in \mathbf{Z}^{d}} g\left(M n+\gamma_{j}\right) e^{i\left(M n+\gamma_{j}\right) \cdot u} \\
& =\sum_{j=0}^{m-1} F_{j}\left(M^{T} u\right) e^{i \gamma_{j} \cdot u}
\end{aligned}
$$

where $F_{j}, j=0,1, \ldots, m-1$, are given by (3.6). By (3.4), $G=J\left(F_{0}, \ldots, F_{m-1}\right)$. Therefore $J$ is surjective, and the inverse of $J$ has the desired form.

For any $G$ in $L^{2}\left(\mathbf{T}^{d}\right)^{q \times r}$, define a function $G_{m o d}$ in $L^{2}\left(\mathbf{T}^{d}\right)^{q \times m r}$ by

$$
G_{m o d}(u)=\frac{1}{\sqrt{m}}\left(G(u) G\left(u+2 \pi\left(M^{T}\right)^{-1} \eta_{1}\right) \ldots G\left(u+2 \pi\left(M^{T}\right)^{-1} \eta_{m-1}\right)\right) .
$$

Remark 3.2. $Q(u)=\Omega_{\text {mod }}(u)$, where $\Omega(u)$ is given by (3.5).

We summarize below some properties of $G_{m o d}$ which will be needed in the next section. We omit their proofs. 
Proposition 3.3. For any $G$ in $L^{2}\left(\mathbf{T}^{d}\right)^{q \times r}$,

(i) $\quad G_{\text {mod }}(u)=\left(J^{*} G\right)\left(M^{T} u\right) Q(u)$,

(ii) $\quad J^{*}(G)(u)=G_{\text {mod }}\left(\left(M^{T}\right)^{-1} u\right) Q\left(\left(M^{T}\right)^{-1} u\right)^{*}$,

(iii) $\frac{1}{m} \sum_{j=0}^{m-1} G\left(u+2 \pi\left(M^{T}\right)^{-1} \eta_{j}\right)=\sum_{n \in \mathbf{Z}^{d}} g(M n) e^{i M n \cdot u}$, where

$$
G(u)=\sum_{n \in \mathbf{Z}^{d}} g(n) e^{i n \cdot u} .
$$

Consider next the special case $q=m r$.

Proposition 3.4. Let $G \in L^{2}\left(\mathbf{T}^{d}\right)^{m r \times r}$. Suppose that the $m r \times m r$ matrices $G_{m o d}(u)$ are invertible for almost all $u$ in $\mathbf{R}^{d}$ and $G_{m o d}^{-1}$ is in $L^{2}\left(\mathbf{T}^{d}\right)^{m r \times m r}$. Let

$$
\tilde{G}(u)=\sqrt{m}\left(G_{m o d}(u)^{-1}\right)^{*}\left(\begin{array}{c}
I_{r} \\
0_{r} \\
\vdots \\
0_{r}
\end{array}\right)_{m r \times r}, u \in \mathbf{R}^{d} .
$$

Then $\tilde{G}$ is in $L^{2}\left(\mathbf{T}^{d}\right)^{m r \times r}$ and $G_{\text {mod }}(u)^{-1}=\tilde{G}_{m o d}(u)^{*}$.

\section{Characterizations of oblique multiwavelets}

We shall follow the same notations as in sections 1 and 3 . As before, let $U=$ $\left(U_{1}, \ldots, U_{d}\right)$ be an ordered $d$-tuple of distinct commuting unitary operators on a Hilbert space $H$. Let $D$ be another unitary operator on $H$ such that

$$
U^{n} D=D U^{M n}, \quad n \in \mathbf{Z}^{d},
$$

where $M$ is a $d \times d$ matrix as in section 3 . Then for every $n$ in $\mathbf{Z}^{d}$, there exist a unique $\ell$ in $\{0,1, \ldots, m-1\}$ and a unique $p$ in $\mathbf{Z}^{d}$ such that $n=M p+\gamma_{\ell}$. Hence

$$
D U^{n}=U^{p} D U^{\gamma_{\ell}} .
$$

Let $X=\left\{x_{1}, \ldots, x_{r}\right\}$ be a finite subset of $H$ such that

$$
\left\{\left\langle x_{j}, U^{n} x_{k}\right\rangle\right\}_{n \in \mathbf{Z}^{d}} \in \ell^{2}\left(\mathbf{Z}^{d}\right), \quad j, k=1, \ldots, r .
$$

Let $L=\{(\ell, j): \ell=0,1, \ldots, m-1, j=1, \ldots, r\}$ with lexicographical ordering in $(\ell, j)$,

$$
y_{\ell, j}=D U^{\gamma_{\ell}} x_{j}, \quad(\ell, j) \in L,
$$

and let $Y=\left\{y_{\ell, j}:(\ell, j) \in L\right\}$. Let

$$
V_{0}=\left\langle U^{\mathbf{Z}^{d}}(X)\right\rangle \quad \text { and } \quad V_{1}=\left\langle U^{\mathbf{Z}^{d}}(Y)\right\rangle .
$$

For the time being, we do not assume that $V_{0} \subset V_{1}$.

By (4.3), the function

$$
\Phi_{X, X}(u)=\left(\sum_{n \in \mathbf{Z}^{d}}\left\langle x_{j}, U^{n} x_{k}\right\rangle e^{i n \cdot u}\right)_{1 \leq j \leq r, 1 \leq k \leq r}
$$


is in $L^{2}\left(\mathbf{T}^{d}\right)^{r \times r}$ and the function

$$
\Phi_{Y, Y}(u)=\left(\sum_{n \in \mathbf{Z}^{d}}\left\langle y_{\ell, j}, U^{n} y_{p, k}\right\rangle e^{i n \cdot u}\right)_{(\ell, j) \in L,(p, k) \in L}
$$

is in $L^{2}\left(\mathbf{T}^{d}\right)^{m r \times m r}$. Define a function $\Phi_{X, X}^{[m]}$ in $L^{2}\left(\mathbf{T}^{d}\right)^{m r \times m r}$ by

$$
\begin{aligned}
& \Phi_{X, X}^{[m]}(u) \\
& =\left(\begin{array}{cccc}
\Phi_{X, X}(u) & & & \\
0_{r} & \Phi_{X, X}\left(u+2 \pi\left(M^{T}\right)^{-1} \eta_{1}\right) & \ddots & 0_{r} \\
\vdots & \ddots & \ddots & \vdots \\
0_{r} & \ldots & 0_{r} & \Phi_{X, X}\left(u+2 \pi\left(M^{T}\right)^{-1} \eta_{m-1}\right)
\end{array}\right) .
\end{aligned}
$$

Theorem 4.1. We have the following relations between $X$ and $Y$.

(i) $\quad V_{1}=D\left(V_{0}\right)$.

(ii) $\quad \Phi_{Y, Y}\left(M^{T} u\right)=Q(u) \Phi_{X, X}^{[m]}(u) Q(u)^{*}$.

(iii) $U^{\mathbf{Z}^{d}}(X)$ is a Riesz basis for $V_{0}$ if and only if $U^{\mathbf{Z}^{d}}(Y)$ is a Riesz basis for $V_{1}$.

Proof. (i) and (iii) follow easily from (4.2) and the assumption that $D$ is unitary.

By (4.1) and (4.4), for $t, p=0,1, \ldots, m-1, j, k=1, \ldots, r$,

$$
\left\langle y_{t, j}, U^{n} y_{p, k}\right\rangle=\left\langle D U^{\gamma_{t}} x_{j}, D U^{M n+\gamma_{p}} x_{k}\right\rangle=\left\langle x_{j}, U^{M n+\gamma_{p}-\gamma_{t}} x_{k}\right\rangle
$$

Hence the $(t, p)$-block of $\Phi_{Y, Y}\left(M^{T} u\right)$ is

$$
\left(\sum_{n \in \mathbf{Z}^{d}}\left\langle y_{t, j}, U^{n} y_{p, k}\right\rangle e^{i n \cdot M^{T} u}\right)_{j, k=1}^{r}=\sum_{n \in \mathbf{Z}^{d}}\left(\left\langle x_{j}, U^{M n+\gamma_{p}-\gamma_{t}} x_{k}\right\rangle\right)_{j, k=1}^{r} e^{i M n \cdot u} .
$$

Using (3.3), the $(t, p)$-block of $Q(u) \Phi_{X, X}^{[m]}(u) Q(u)^{*}$ is

$$
\begin{aligned}
\left(^{*}\right) & =\frac{1}{m} \sum_{\ell=0}^{m-1} e^{i\left(\gamma_{t} \cdot u+2 \pi \eta_{\ell} \cdot M^{-1} \gamma_{t}\right)} \Phi_{X, X}\left(u+2 \pi\left(M^{T}\right)^{-1} \eta_{\ell}\right) e^{-i\left(\gamma_{p} \cdot u+2 \pi \eta_{\ell} \cdot M^{-1} \gamma_{p}\right)} \\
& =\frac{1}{m} \sum_{\ell=0}^{m-1} G\left(u+2 \pi\left(M^{T}\right)^{-1} \eta_{\ell}\right)
\end{aligned}
$$

where

$$
\begin{aligned}
G(u) & =\Phi_{X, X}(u) e^{i\left(\gamma_{t}-\gamma_{p}\right) \cdot u}=\left(\sum_{n \in \mathbf{Z}^{d}}\left\langle x_{j}, U^{n} x_{k}\right\rangle e^{i\left(n+\gamma_{t}-\gamma_{p}\right) \cdot u}\right)_{j, k=1}^{r} \\
& =\sum_{n \in \mathbf{Z}^{d}}\left(\left\langle x_{j}, U^{n+\gamma_{p}-\gamma_{t}} x_{k}\right\rangle\right)_{j, k=1}^{r} e^{i n \cdot u} .
\end{aligned}
$$

Hence by Proposition 3.3

$$
\left(^{*}\right)=\sum_{n \in \mathbf{Z}^{d}}\left(\left\langle x_{j}, U^{M n+\gamma_{p}-\gamma_{t}} x_{k}\right\rangle\right)_{j, k=1}^{r} e^{i M n \cdot u},
$$

which is the $(t, p)$-block of $\Phi_{Y, Y}\left(M^{T} u\right)$. Hence (ii) holds. 
Henceforth, assume that $U^{\mathbf{Z}^{d}}(X)$ is a Riesz basis for $V_{0}$ so that $U^{\mathbf{Z}^{d}}(Y)$ is a Riesz basis for $V_{1}=D\left(V_{0}\right)$. Let $\widetilde{X}=\left\{\tilde{x}_{1}, \ldots, \tilde{x}_{r}\right\}$ be a subset of $V_{0}$ such that $U^{\mathbf{Z}^{d}}(\widetilde{X})$ is a Riesz basis for $V_{0}$ biorthogonal to $U^{\mathbf{Z}^{d}}(X)$. Since $D$ is unitary, $\left\{D U^{n} x_{j}: n \in\right.$ $\left.\mathbf{Z}^{d}, j=1, \ldots, r\right\}$ and $\left\{D U^{n} \tilde{x}_{j}: n \in \mathbf{Z}^{d}, j=1, \ldots, r\right\}$ are biorthogonal Riesz bases for $V_{1}$. Let

$$
\tilde{y}_{\ell, j}=D U^{\gamma_{\ell}} \tilde{x}_{j}, \quad(\ell, j) \in L,
$$

and let $\widetilde{Y}=\left\{\tilde{y}_{\ell, j}:(\ell, j) \in L\right\}$. It is not difficult to show that $U^{\mathbf{Z}^{d}}(Y)$ and $U^{\mathbf{Z}^{d}}(\widetilde{Y})$ are biorthogonal Riesz bases for $V_{1}$.

Let $W=\left\{w_{1}, \ldots, w_{q}\right\}$ be a subset of $V_{1}$, for some positive integer $q$. Then

$$
w_{k}=\sum_{j=1}^{r} \sum_{n \in \mathbf{Z}^{d}} g_{k, j}(n) D U^{n} x_{j}, \quad k=1, \ldots, q,
$$

where $g_{k, j} \in \ell^{2}\left(\mathbf{Z}^{d}\right), k=1, \ldots, q, j=1, \ldots, r$. Let

$$
g(n)=\left(g_{k, j}(n)\right)_{1 \leq k \leq q, 1 \leq j \leq r}, \quad n \in \mathbf{Z}^{d} .
$$

Then $g$ is in $\ell^{2}\left(\mathbf{Z}^{d}\right)^{q \times r}$ and (4.10) can be written in matrix form as

$$
\left(\begin{array}{c}
w_{1} \\
\vdots \\
w_{q}
\end{array}\right)=\sum_{n \in \mathbf{Z}^{d}} g(n)\left(\begin{array}{c}
D U^{n} x_{1} \\
\vdots \\
D U^{n} x_{r}
\end{array}\right) .
$$

Define a function $G$ in $L^{2}\left(\mathbf{T}^{d}\right)^{q \times r}$ by

$$
G(u)=\sum_{n \in \mathbf{Z}^{d}} g(n) e^{i n \cdot u}, \quad u \in \mathbf{R}^{d} .
$$

Theorem 4.2. The function $G$ associated with $W$ has the following properties.

(i) $\Phi_{W, \tilde{Y}}(u)=J^{*}(G)(u)$.

(ii) $\quad G_{\text {mod }}(u)=\Phi_{W, \tilde{Y}}\left(M^{T} u\right) Q(u)$.

(iii) $U^{\mathbf{Z}^{d}}(W)$ is a Riesz basis for $\left\langle U^{\mathbf{Z}^{d}}(W)\right\rangle$ if and only if there exist positive constants $C_{1}$ and $C_{2}$ such that

$$
C_{1} \leq G_{\text {mod }}(u) G_{\text {mod }}(u)^{*} \leq C_{2} \quad \text { a.e. }
$$

Proof. (i): By (4.10), (4.2), (4.1) and (4.4), for $k=1, \ldots, q$,

$$
\begin{aligned}
w_{k} & =\sum_{j=1}^{r} \sum_{n \in \mathbf{Z}^{d}} \sum_{\ell=0}^{m-1} g_{k, j}\left(M n+\gamma_{\ell}\right) D U^{M n+\gamma_{\ell}} x_{j} \\
& =\sum_{\ell=0}^{m-1} \sum_{j=1}^{r} \sum_{n \in \mathbf{Z}^{d}} g_{k, j}\left(M n+\gamma_{\ell}\right) U^{n} y_{\ell, j} .
\end{aligned}
$$

Therefore for $\ell=0,1, \ldots, m-1$ and $j=1, \ldots, r$,

$$
\sum_{n \in \mathbf{Z}^{d}}\left\langle w_{k}, U^{n} \tilde{y}_{\ell, j}\right\rangle e^{i n \cdot u}=\sum_{n \in \mathbf{Z}^{d}} g_{k, j}\left(M n+\gamma_{\ell}\right) e^{i n \cdot u} .
$$


Hence by Theorem 3.1 ,

$$
\begin{aligned}
\Phi_{W, \widetilde{Y}}(u) & =\left(\sum_{n \in \mathbf{Z}^{d}} g(M n) e^{i n \cdot u} \sum_{n \in \mathbf{Z}^{d}} g\left(M n+\gamma_{1}\right) e^{i n \cdot u} \cdots \sum_{n \in \mathbf{Z}^{d}} g\left(M n+\gamma_{m-1}\right) e^{i n \cdot u}\right) \\
& =J^{*}(G)(u) .
\end{aligned}
$$

(ii) follows from (i) and Proposition 3.3 ,

By [10 Proposition 3.4], $U^{\mathbf{Z}^{d}}(W)$ is a Riesz basis for $\left\langle U^{\mathbf{Z}^{d}}(W)\right\rangle$ if and only if there exist positive constants $C_{1}$ and $C_{2}$ such that

$$
C_{1} \leq \Phi_{W, \tilde{Y}}(u) \Phi_{W, \tilde{Y}}(u)^{*} \leq C_{2} \quad \text { a.e. }
$$

Then (iii) follows from the relation

$$
G_{m o d}(u) G_{m o d}(u)^{*}=\Phi_{W, \widetilde{Y}}(u) \Phi_{W, \widetilde{Y}}(u)^{*},
$$

which is obvious from (ii).

We shall now see the effect of the change of bases for $V_{1}$.

Corollary 4.3. Let a vector $y$ in $V_{1}$ be expressed as

$$
y=\sum_{j=1}^{r} \sum_{n \in \mathbf{Z}^{d}} a_{j}(n) D U^{n} x_{j}=\sum_{\ell=1}^{m-1} \sum_{j=1}^{r} \sum_{n \in \mathbf{Z}^{d}} c_{\ell, j}(n) U^{n} y_{\ell, j},
$$

where $a_{j}$ and $c_{\ell, j}$ are in $\ell^{2}\left(\mathbf{Z}^{d}\right), j=1, \ldots, r, \ell=0,1, \ldots, m-1$. Let

$$
\begin{aligned}
A_{j}(u) & =\sum_{n \in \mathbf{Z}^{d}} a_{j}(n) e^{i n \cdot u}, \quad j=1, \ldots, r, \\
C_{\ell, j}(u) & =\sum_{n \in \mathbf{Z}^{d}} c_{\ell, j}(n) e^{i n \cdot u}, \quad \ell=0,1, \ldots, m-1, j=1, \ldots, r,
\end{aligned}
$$

and

$$
A=\left(A_{1} \ldots A_{r}\right), \quad C=\left(\left(C_{0, j}\right)_{j=1, \ldots, r} \ldots\left(C_{m-1, j}\right)_{j=1, \ldots, r}\right) .
$$

Then $C=J^{*}(A)$.

Proof. By Theorem 4.2(i), $C(u)=\Phi_{\{y\}, \widetilde{Y}}(u)=J^{*}(A)(u)$.

Corollary 4.4. Let $W=\left\{w_{1}, \ldots, w_{q}\right\}$ and $\Gamma=\left\{z_{1}, \ldots, z_{p}\right\}$ be subsets of $V_{1}$ such that $\left\{\left\langle w_{k}, U^{n} z_{j}\right\rangle\right\}_{n \in \mathbf{Z}^{d}} \in \ell^{2}\left(\mathbf{Z}^{d}\right), \quad k=1, \ldots, q, j=1, \ldots, p$, and

$$
\left(\begin{array}{c}
w_{1} \\
\vdots \\
w_{q}
\end{array}\right)=\sum_{n \in \mathbf{Z}^{d}} g(n)\left(\begin{array}{c}
D U^{n} x_{1} \\
\vdots \\
D U^{n} x_{r}
\end{array}\right), \quad\left(\begin{array}{c}
z_{1} \\
\vdots \\
z_{p}
\end{array}\right)=\sum_{n \in \mathbf{Z}^{d}} f(n)\left(\begin{array}{c}
D U^{n} x_{1} \\
\vdots \\
D U^{n} x_{r}
\end{array}\right),
$$

where $g$ is in $\ell^{2}\left(\mathbf{Z}^{d}\right)^{q \times r}$ and $f$ is in $\ell^{2}\left(\mathbf{Z}^{d}\right)^{p \times r}$. Define

$$
G(u)=\sum_{n \in \mathbf{Z}^{d}} g(n) e^{i n \cdot u}, \quad F(u)=\sum_{n \in \mathbf{Z}^{d}} f(n) e^{i n \cdot u}, u \in \mathbf{R}^{d} .
$$

Then

$$
\Phi_{W, \Gamma}\left(M^{T} u\right)=G_{m o d}(u) \Phi_{X, X}^{[m]}(u) F_{\text {mod }}(u)^{*}
$$

$$
=\frac{1}{m} \sum_{j=0}^{m-1} G\left(u+2 \pi\left(M^{T}\right)^{-1} \eta_{j}\right) \Phi_{X, X}\left(u+2 \pi\left(M^{T}\right)^{-1} \eta_{j}\right) F\left(u+2 \pi\left(M^{T}\right)^{-1} \eta_{j}\right)^{*} .
$$


If moreover $U^{\mathbf{Z}^{d}}(X)$ is orthonormal, then

(i) $\Phi_{W, \Gamma}\left(M^{T} u\right)=G_{\text {mod }}(u) F_{\text {mod }}(u)^{*}$;

(ii) assuming $\left\{\left\langle w_{k}, U^{n} w_{j}\right\rangle\right\}_{n \in \mathbf{Z}^{d}} \in \ell^{2}\left(\mathbf{Z}^{d}\right), \quad k, j=1, \ldots, q$, then $U^{\mathbf{Z}^{d}}(W)$ is orthonormal if and only if $G_{\text {mod }}(u) G_{m o d}(u)^{*}=I_{q}$ a.e.

Proof. By [10, Proposition 3.3], Theorem 4.1] and Theorem 4.2.

$$
\begin{aligned}
\Phi_{W, \Gamma}\left(M^{T} u\right) & =\Phi_{W, \tilde{Y}}\left(M^{T} u\right) \Phi_{Y, Y}\left(M^{T} u\right) \Phi_{\Gamma, \tilde{Y}}\left(M^{T} u\right)^{*} \\
& =\Phi_{W, \tilde{Y}}\left(M^{T} u\right) Q(u) \Phi_{X, X}^{[m]}(u) Q(u)^{*} \Phi_{\Gamma, \tilde{Y}}\left(M^{T} u\right)^{*} \\
& =G_{m o d}(u) \Phi_{X, X}^{[m]}(u) F_{m o d}(u)^{*}
\end{aligned}
$$

The rest of the assertions are straightforward.

We now apply our previous results to the special case when $V_{0} \subset V_{1}$. The next theorem, which is a consequence of Theorem 2.1 and Theorem 4.2, gives characterizations of oblique multiwavelets in the general setting of a Hilbert space.

Theorem 4.5. Assume that

$$
V_{0} \subset V_{1} .
$$

Let $p=m r-r, \Gamma=\left\{z_{1}, \ldots, z_{p}\right\} \subset V_{1} \backslash V_{0}$,

$$
\left(\begin{array}{c}
x_{1} \\
\vdots \\
x_{r}
\end{array}\right)=\sum_{n \in \mathbf{Z}^{d}} h(n)\left(\begin{array}{c}
D U^{n} x_{1} \\
\vdots \\
D U^{n} x_{r}
\end{array}\right), \quad\left(\begin{array}{c}
z_{1} \\
\vdots \\
z_{p}
\end{array}\right)=\sum_{n \in \mathbf{Z}^{d}} g(n)\left(\begin{array}{c}
D U^{n} x_{1} \\
\vdots \\
D U^{n} x_{r}
\end{array}\right),
$$

where $h$ is in $\ell^{2}\left(\mathbf{Z}^{d}\right)^{r \times r}$ and $g$ is in $\ell^{2}\left(\mathbf{Z}^{d}\right)^{p \times r}$. Define

$$
H(u)=\sum_{n \in \mathbf{Z}^{d}} h(n) e^{i n \cdot u}, G(u)=\sum_{n \in \mathbf{Z}^{d}} g(n) e^{i n \cdot u}, u \in \mathbf{R}^{d} .
$$

Let $S=X \cup \Gamma$ and

$$
F(u)=\left(\begin{array}{c}
H(u) \\
G(u)
\end{array}\right), u \in \mathbf{R}^{d} .
$$

The following conditions are equivalent:

(i) $\quad U^{\mathbf{Z}^{d}}(S)$ is a Riesz basis for $V_{1}$.

(ii) $\quad U^{\mathbf{Z}^{d}}(\Gamma)$ is a Riesz basis for $W_{0}:=\left\langle U^{\mathbf{Z}^{d}}(\Gamma)\right\rangle$ and $V_{0} \oplus W_{0}=V_{1}$.

(iii) There exist positive constants $C_{1}$ and $C_{2}$ such that

$$
C_{1} \leq F_{\text {mod }}(u) F_{\text {mod }}(u)^{*} \leq C_{2} \quad \text { a.e. }
$$

(iv) The matrices $F_{\text {mod }}(u)$ are invertible for almost all $u$, and the functions $u \longrightarrow$ $\left\|F_{\text {mod }}(u)\right\|$ and $u \longrightarrow\left\|F_{\text {mod }}(u)^{-1}\right\|$ are essentially bounded.

(v) The operator $R: L^{2}\left(\mathbf{T}^{d}\right)^{r} \oplus L^{2}\left(\mathbf{T}^{d}\right)^{p} \longrightarrow L^{2}\left(\mathbf{T}^{d}\right)^{r}$ defined by

$$
\begin{aligned}
R(A, B)(u) & =\left(A\left(M^{T} u\right) B\left(M^{T} u\right)\right)\left(\begin{array}{c}
H(u) \\
G(u)
\end{array}\right) \\
& =A\left(M^{T} u\right) H(u)+B\left(M^{T} u\right) G(u)
\end{aligned}
$$

is bounded and invertible. 
Proof. By Theorem 4.2,

$$
\begin{gathered}
\Phi_{S, \tilde{Y}}(u)=J^{*}(F)(u), \\
F_{\text {mod }}(u)=\Phi_{S, \tilde{Y}}\left(M^{T} u\right) Q(u),
\end{gathered}
$$

and

$$
F_{\text {mod }}(u) F_{\text {mod }}(u)^{*}=\Phi_{S, \tilde{Y}}\left(M^{T} u\right) \Phi_{S, \tilde{Y}}\left(M^{T} u\right)^{*} .
$$

Next, by composing the operator $R_{0}: L^{2}\left(\mathbf{T}^{d}\right)^{r} \oplus L^{2}\left(\mathbf{T}^{d}\right)^{p} \longrightarrow L^{2}\left(\mathbf{T}^{d}\right)^{m r}$ defined in (2.4) with the unitary operator $J: L^{2}\left(\mathbf{T}^{d}\right)^{m r} \longrightarrow L^{2}\left(\mathbf{T}^{d}\right)^{r}$ defined in (3.4) with $q=1$, and using 4.18,

$$
\begin{aligned}
J R_{0}(A, B)(u) & =\left(A\left(M^{T} u\right) B\left(M^{T} u\right)\right)\left(\begin{array}{c}
\Phi_{X, \tilde{Y}}\left(M^{T} u\right) \\
\Phi_{\Gamma, \tilde{Y}}\left(M^{T} u\right)
\end{array}\right) \Omega(u) \\
& =\left(A\left(M^{T} u\right) B\left(M^{T} u\right)\right) F(u)=R(A, B)(u) .
\end{aligned}
$$

Hence

$$
R=J R_{0}
$$

By (4.20), (4.19) and 4.21), the equivalence of (i) to (v) in this theorem then follows easily from that in Theorem 2.1.

Remark 4.6. Suppose that $U^{\mathbf{Z}^{d}}(X)$ is orthonormal. Then $U^{\mathbf{Z}^{d}}(S)$ is orthonormal if and only if $F_{\text {mod }}(u)$ are unitary for almost all $u \in \mathbf{R}^{d}$.

The next two results are analogues of Proposition 2.2 and Theorem 2.4 respectively.

Proposition 4.7. Suppose that the conditions in Theorem 4.5 hold and $p=m r-r$. Let $\tilde{F} \in L^{2}\left(\mathbf{T}^{d}\right)^{m r \times r}$ be given as in Proposition 3.4 such that

$$
\tilde{F}_{\text {mod }}(u)^{*}=F_{\text {mod }}(u)^{-1} .
$$

(i) Then $R^{-1}: L^{2}\left(\mathbf{T}^{d}\right)^{r} \longrightarrow L^{2}\left(\mathbf{T}^{d}\right)^{r} \oplus L^{2}\left(\mathbf{T}^{d}\right)^{p}=L^{2}\left(\mathbf{T}^{d}\right)^{m r}$ is given by

$$
(4.23) R^{-1}(C)\left(M^{T} u\right)=C_{\text {mod }}(u) \tilde{F}_{\text {mod }}(u)^{*}
$$

$$
=\frac{1}{m} \sum_{j=0}^{m-1} C\left(u+2 \pi\left(M^{T}\right)^{-1} \eta_{j}\right) \tilde{F}\left(u+2 \pi\left(M^{T}\right)^{-1} \eta_{j}\right)^{*} .
$$

(ii) Write

$\tilde{F}(u)=\left(\begin{array}{c}\tilde{H}(u) \\ \tilde{G}(u)\end{array}\right)$, where $\tilde{H}(u)=\sum_{n \in \mathbf{Z}^{d}} \tilde{h}(n) e^{i n \cdot u}, \tilde{G}(u)=\sum_{n \in \mathbf{Z}^{d}} \tilde{g}(n) e^{i n \cdot u}, u \in \mathbf{R}^{d}$,

$\tilde{h}$ is in $\ell^{2}\left(\mathbf{Z}^{d}\right)^{r \times r}$ and $\tilde{g}$ is in $\ell^{2}\left(\mathbf{Z}^{d}\right)^{p \times r}$. Let $X^{\prime}=\left\{x_{1}^{\prime}, \ldots, x_{r}^{\prime}\right\}$ and $\Gamma^{\prime}=\left\{z_{1}^{\prime}, \ldots, z_{p}^{\prime}\right\}$ be subsets of $V_{1}$ defined by

$$
\left(\begin{array}{c}
x_{1}^{\prime} \\
\vdots \\
x_{r}^{\prime}
\end{array}\right)=\sum_{n \in \mathbf{Z}^{d}} \tilde{h}(n)\left(\begin{array}{c}
D U^{n} \tilde{x}_{1} \\
\vdots \\
D U^{n} \tilde{x}_{r}
\end{array}\right), \quad\left(\begin{array}{c}
z_{1}^{\prime} \\
\vdots \\
z_{p}^{\prime}
\end{array}\right)=\sum_{n \in \mathbf{Z}^{d}} \tilde{g}(n)\left(\begin{array}{c}
D U^{n} \tilde{x}_{1} \\
\vdots \\
D U^{n} \tilde{x}_{r}
\end{array}\right),
$$

and let $\widetilde{S}=X^{\prime} \cup \Gamma^{\prime}$. Then $U^{\mathbf{Z}^{d}}(\widetilde{S})$ is a Riesz basis for $V_{1}$ biorthogonal to $U^{\mathbf{Z}^{d}}(S)$, $U^{\mathbf{Z}^{d}}\left(X^{\prime}\right)$ biorthogonal to $U^{\mathbf{Z}^{d}}(X), U^{\mathbf{Z}^{d}}\left(\Gamma^{\prime}\right)$ biorthogonal to $U^{\mathbf{Z}^{d}}(\Gamma), U^{\mathbf{Z}^{d}}\left(X^{\prime}\right)$ $\perp U^{\mathbf{Z}^{d}}(\Gamma)$ and $U^{\mathbf{Z}^{d}}\left(\Gamma^{\prime}\right) \perp U^{\mathbf{Z}^{d}}(X)$. 
Proof. (i): Since $R=J R_{0}$, by Proposition 3.3, (4.19) and (4.22),

$$
\begin{aligned}
R^{-1}(C)(u) & =R_{0}^{-1}\left(J^{*} C\right)(u) \\
& =C_{\text {mod }}\left(\left(M^{T}\right)^{-1} u\right) Q\left(\left(M^{T}\right)^{-1} u\right)^{*} \Phi_{S, \tilde{Y}}(u)^{-1} \\
& =C_{\text {mod }}\left(\left(M^{T}\right)^{-1} u\right) F_{\text {mod }}\left(\left(M^{T}\right)^{-1} u\right)^{-1} \\
& =C_{\text {mod }}\left(\left(M^{T}\right)^{-1} u\right) \tilde{F}_{\text {mod }}\left(\left(M^{T}\right)^{-1} u\right)^{*}
\end{aligned}
$$

(ii): By Theorem 4.2 and Theorem 4.5 (with $X$ and $Y$ there replaced by $\widetilde{X}$ and $\tilde{Y}$, respectively), $U^{\mathbf{Z}^{d}}(\widetilde{S})$ is a Riesz basis for $V_{1}$ and

$$
\tilde{F}_{\text {mod }}(u)=\Phi_{\widetilde{S}, Y}\left(M^{T} u\right) Q(u) .
$$

By [10, Proposition 3.3], (4.19), (4.24) and (4.22),

$$
\Phi_{S, \widetilde{S}}(u)=\Phi_{S, \widetilde{Y}}(u) \Phi_{\widetilde{S}, Y}(u)^{*}=F_{\text {mod }}\left(\left(M^{T}\right)^{-1} u\right) \tilde{F}_{\text {mod }}\left(\left(M^{T}\right)^{-1} u\right)^{*}=I_{m r} \text { a.e. }
$$

Hence $U^{\mathbf{Z}^{d}}(\widetilde{S})$ is biorthogonal to $U^{\mathbf{Z}^{d}}(S)$. The rest of the assertions are obvious.

Theorem 4.8. Suppose that the conditions in Theorem 4.5 hold. Let $p=m r-r$,

$$
\begin{aligned}
& A_{j}(u)=\sum_{n \in \mathbf{Z}^{d}} a_{j}(n) e^{i n \cdot u}, \quad a_{j} \in \ell^{2}\left(\mathbf{Z}^{d}\right), \quad j=1, \ldots, r, \\
& B_{k}(u)=\sum_{n \in \mathbf{Z}^{d}} b_{k}(n) e^{i n \cdot u}, \quad b_{k} \in \ell^{2}\left(\mathbf{Z}^{d}\right), \quad k=1, \ldots, p, \\
& P_{j}(u)=\sum_{n \in \mathbf{Z}^{d}} p_{j}(n) e^{i n \cdot u}, \quad p_{j} \in \ell^{2}\left(\mathbf{Z}^{d}\right), \quad j=1, \ldots, r,
\end{aligned}
$$

and

$$
A=\left(A_{1} \ldots A_{r}\right), \quad B=\left(B_{1} \ldots B_{p}\right), \quad P=\left(P_{1} \ldots P_{r}\right) .
$$

The following conditions are equivalent:

$$
\sum_{j=1}^{r} \sum_{n \in \mathbf{Z}^{d}} p_{j}(n) D U^{n} x_{j}=\sum_{j=1}^{r} \sum_{n \in \mathbf{Z}^{d}} a_{j}(n) U^{n} x_{j}+\sum_{k=1}^{p} \sum_{n \in \mathbf{Z}^{d}} b_{k}(n) U^{n} z_{k} .
$$

$$
\text { (Reconstruction algorithm) }
$$

$$
=A\left(M^{T} u\right) H(u)+B\left(M^{T} u\right) G(u) .
$$

(iii) (Decomposition algorithm)

$$
\begin{gathered}
(A(u), B(u))=R^{-1}(P)(u) \\
=\frac{1}{m} \sum_{j=0}^{m-1} P\left(\left(M^{T}\right)^{-1}\left(u+2 \pi \eta_{j}\right)\right) \tilde{F}\left(\left(M^{T}\right)^{-1}\left(u+2 \pi \eta_{j}\right)\right)^{*},
\end{gathered}
$$

where $\tilde{F}$ is given in (4.22).

Proof. Write

$$
\sum_{j=1}^{r} \sum_{n \in \mathbf{Z}^{d}} p_{j}(n) D U^{n} x_{j}=\sum_{\ell=1}^{m-1} \sum_{j=1}^{r} \sum_{n \in \mathbf{Z}^{d}} c_{\ell, j}(n) U^{n} y_{\ell, j},
$$


where $c_{\ell, j}$ are in $\ell^{2}\left(\mathbf{Z}^{d}\right), j=1, \ldots, r, \ell=0,1, \ldots, m-1$. Let

$$
C_{\ell, j}(u)=\sum_{n \in \mathbf{Z}^{d}} c_{\ell, j}(n) e^{i n \cdot u}, \quad \ell=0,1, \ldots, m-1, j=1, \ldots, r,
$$

and $C=\left(\left(C_{0, j}\right)_{j=1, \ldots, r} \ldots\left(C_{m-1, j}\right)_{j=1, \ldots, r}\right)$. By Corollary $4.3 \quad C=J^{*}(P)$. Then by Theorem 2.4,

$$
\text { (i) } \begin{aligned}
& \Longleftrightarrow C=R_{0}(A, B) \\
& \Longleftrightarrow J^{*}(P)=R_{0}(A, B) \\
& \Longleftrightarrow P=J R_{0}(A, B)=R(A, B) \\
& \Longleftrightarrow(A, B)=R^{-1}(P) .
\end{aligned}
$$

Note that we can also obtain formulae for the projections $P_{V_{0} / / W_{0}}$ and $P_{W_{0} / / V_{0}}$, as in Corollary 2.5. We omit the details here.

Remark 4.9. (i) The results in this paper can be applied to the special case when $H=L^{2}\left(\mathbf{R}^{d}\right),\left(U_{k} f\right)(x)=f\left(x-e_{k}\right), k=1, \ldots, d$, and $(D f)(x)=m^{\frac{1}{2}} f(M x)$, for $x$ in $\mathbf{R}^{d}$ and $f$ in $L^{2}\left(\mathbf{R}^{d}\right)$, where $e_{k}=\left(\delta_{k, j}\right)_{j=1, \ldots, d}, k=1, \ldots, d, M$ is a $d \times d$ dilation matrix considered in section 3 and $m=|\operatorname{det}(M)| \geq 2$. In this case,

$$
\left(D U^{n} f\right)(x)=m^{\frac{1}{2}} f(M x-n), \quad n \in \mathbf{Z}^{d},
$$

and if $V=\left\{f_{1}, \ldots, f_{r}\right\}$ and $W=\left\{g_{1}, \ldots, g_{p}\right\}$ are subsets of $L^{2}\left(\mathbf{R}^{d}\right)$, then

$$
\Phi_{V, W}(u)=\left(\sum_{n \in \mathbf{Z}^{d}} \hat{f}_{k}(u+2 \pi n) \overline{\hat{g}_{j}(u+2 \pi n)}\right)_{1 \leq k \leq r, 1 \leq j \leq p}
$$

where $\hat{f}$ is the Fourier transform of a function $f$ in $L^{2}\left(\mathbf{R}^{d}\right)$.

(ii) If $d=1$, and $U$ and $D$ are unitary operators on a Hilbert space $H$ such that $U D=D U^{2}$, using the notations in Theorem 4.5, then

$$
F_{\text {mod }}(u)=\frac{1}{\sqrt{2}}\left(\begin{array}{cc}
H(u) & H(u+\pi) \\
G(u) & G(u+\pi)
\end{array}\right) .
$$

In particular, Theorem 4.5 generalizes the characterizations of oblique multiwavelets given in [1, Theorem 3.1] and [2, Proposition 3.1, Theorem 3.2, Corollary 3.4], where the setting $H=L^{2}(\mathbf{R}),(U f)(x)=f(x-1)$ and $(D f)(x)=\sqrt{2} f(2 x)$ is considered.

\section{ACKNOWLEDGEMENT}

This research was supported by the Wavelets Strategic Research Programme, National University of Singapore, under a grant from the National Science and Technology Board and the Ministry of Education, Republic of Singapore. We would like to thank Professor S. L. Lee for his helpful comments on this paper.

\section{REFERENCES}

1. A. Aldroubi, Oblique and hierarchical multiwavelet bases, Appl. Comput. Harmonic Anal. 4 (1997), 231-263. MR 98k:42037

2. A. Aldroubi and M. Papadakis, Characterization and parametrization of multiwavelet bases, Contemporary Math. (1998). MR 99b:42010

3. A. Cohen, I. Daubechies and J. C. Feauveau, Biorthogonal bases of compactly supported wavelets, Comm. Pure Appl. Math. XLV (1992), 485-560. MR 93e:42044 
4. T. N. T. Goodman, S. L. Lee and W. S. Tang, Wavelets in wandering subspaces, Trans. Amer. Math. Soc. 338 (1993), 639-654. MR 93j:42017

5. T. N. T. Goodman, S. L. Lee and W. S. Tang, Wavelet bases for a set of commuting unitary operators, Adv. Comput. Math. 1 (1993), 109-126. MR 94h:42057

6. R. Q. Jia and Z. W. Shen, Multiresolution and wavelets, Proc. Edinburgh Math. Soc. 37 (1994), 271-300. MR 95h:42035

7. S. L. Lee, H. H. Tan and W. S. Tang, Wavelet bases for a unitary operator, Proc. Edinburgh Math. Soc. 38 (1995), 233-260. MR 96g:42019

8. S. L. Lee and W. S. Tang, Characterizations of wavelet bases and frames in Hilbert spaces, Proc. SPIE. 3169 (1997), 282-290.

9. M. A. Naimark, Normed Rings, Wolters-Noordhoff Publishing, Groningen, 1970. MR 50:8075

10. W. S. Tang, Oblique projections, biorthogonal Riesz bases and multiwavelets in Hilbert spaces, Proc. Amer. Math. Soc. (to appear). CMP 98:14

11. R. M. Young, An Introduction to Nonharmonic Fourier Series, Academic Press, New York, 1980. MR 81m:42027

Department of Mathematics, National University of Singapore, 10 Kent Ridge CresCent, 119260, Republic of Singapore

E-mail address: mattws@math.nus.edu.sg 